nature

immunology

\title{
Borrowing words, or claiming them?
}

\author{
Journals are taking steps to stem of the practice of plagiarism.
}

$\mathrm{H}$ ave you ever experienced a sense of déjà $v u$ after reading a colleague's manuscript or researching a topic of interest? A paragraph or entire section sounds eerily familiar - too familiar, perhaps, because it is a word-for-word, verbatim (or nearly so, with a few synonyms tossed in) replication of another piece written by different authors. Or maybe a result or hypothesis is claimed to be 'novel' but in fact others have reported such findings and the previous work is not cited. Or the same data are presented in both earlier and subsequent publications from an author, but the later publication fails to acknowledge the fact that the data were included in the earlier work. Are any of these situations acceptable? In fact they are not. All three scenarios represent examples of what can be considered plagiarism.

Like falsification and fabrication of data, plagiarism is considered an act of scientific misconduct by most institutions and journals. The US Office of Research Integrity (ORI) defines plagiarism as "the appropriation of another person's ideas, processes, results, or words without giving appropriate credit." A 2006 White Paper published by the Council of Science Editors (http://www.councilscienceeditors. org/editorial_policies/white_paper.cfm) states that the reuse of previously published figures or tables, or extensive overlap with the textual findings or analyses of another publication without proper citation, is considered duplicate publication and may constitute copyright infringement of the original piece. In severe cases, authors might be asked to retract the paper in question. If detected before publication, plagiarism can be grounds for rejection of a manuscript under consideration. These policies are clearly expressed in the Nature guide to authors (http:// www.nature.com/authors/gta.pdf) and apply to all Nature-branded journals.

Host institutions or, if the research is funded in the US by the Public Health Service, the ORI may initiate their own investigation. Findings that arise from such investigation could clear the author(s) of any wrongdoing or, if wrongdoing is acknowledged or sufficient evidence is found to suggest that misconduct with the intent to deceive did occur, the institution (or governmental regulatory agency) might take further disciplinary action against the person(s) involved. The ORI details in annual reports (http://ori.dhhs.gov/documents/annual_reports/ori_ annual_report_2007.pdf) the number of alleged cases of misconduct under investigation and publishes in the Federal Register the names and offenses of those people sanctioned after their investigation closes. Authors found guilty of plagiarizing content may be banned from receiving future federal funding. Clearly, the consequences of being caught can lead to severe damage to one's scientific career.

Estimates on the prevalence of plagiarized material vary, but the volume of content published has exploded since the creation of the Internet, and the ease of accessibility of online information may prove too tempting to those who want to take shortcuts. The worry that plagiarism and other forms of scientific misconduct are increasing has prompted many research institutions and governmental agencies to develop guidelines for investigating allegations of scientific 'fraud' and to institute research ethics programs that promote good scientific conduct. In 2007, the ORI and European Science Foundation cosponsored the first World Conference on Research Integrity, held in Lisbon, Portugal, to discuss the implementation of institutional policies to educate researchers about potentially fraudulent practices and to install regulatory oversight mechanisms to investigate accusations of scientific misconduct, including plagiarism. The success of this forum can be appreciated by the number of member states that are moving to set up regulatory bodies and establish requirements for educational and research institutes to develop programs to deal with such cases (http://www.esf.org/publications/corporate-publications. $\mathrm{html}$ ). A second such forum on research integrity is slated for 2010 in Singapore.

Publishers, too, are taking steps to combat the rise of plagiarism and to protect the intellectual property rights of their authors. Internetbased search tools have been developed to detect potential cases of plagiarism. Online publication has also facilitated the creation of a textual database, called 'CrossRef', where published content can be deposited and annotated by various 'meta tags'. Nature Publishing Group and over 40 other publishers, as participating members of CrossRef, routinely deposit published papers into this database. In 2008, the developers of CrossRef also launched a service called 'CrossCheck', which uses the iThenticate Internet-based tool developed by iParadigms to compare a selected paper with the entire database to assess textual similarity. Akin to a search of a protein or nucleic acid database, textual similarity scores are reported after the search program is run, and the context of the similarity can be displayed so that the user (in this case, an editor) can further inspect those manuscripts deemed 'suspect'. The incorporation of such tools into the normal editorial workflow should help diminish the likelihood of plagiarism in manuscripts that might otherwise have passed peer review. Nature Publishing Group journals will be using this tool to scrutinize manuscripts selected for publication.

Still, the onus is on mentors and laboratory chiefs to serve as examples of good scientific conduct. They should initiate discussions about what constitutes plagiarism and 'self plagiarism', as well as other forms of misconduct, with their trainees. Mentors should recognize their obligation to help trainees to develop and hone good written communication skills that follow high ethical standards. Likewise, colleagues, referees and editors all must accept their responsibility to safeguard scientific literature against the possibility of plagiarism or dual publication. Scientific integrity includes the ability to acknowledge good ideas and to give proper credit due to original authors. 